\title{
Towards a Supportive City with Smart Urban Objects in the Internet of Things: The Case of Adaptive Park Bench and Adaptive Light
}

\author{
Marvin Hubl \\ Chair of Information Systems II, \\ University of Hohenheim, \\ Stuttgart, Germany \\ Email: marvin.hubl@uni-hohenheim.de
}

\author{
Philipp Skowron, Michael Aleithe \\ Chair of Information Management \\ Leipzig University, \\ Leipzig, Germany \\ Email: \{skowron, aleithe\}@wifa.uni-leipzig.de
}

\begin{abstract}
IoT technology is a key driver for building smart city infrastructure. The potentials for urban management problems requiring process control and allocation mechanisms has long been acknowledged. However, up to now the potentials of equipping urban objects with sensors, information processing capability, and actuators to enable adaptation to pedestrians' individual needs have not yet been exploited. The objective of our research is to design smart urban objects that enhance usability and safety of the urban space for pedestrians. We report on our conceptual design for an IoT platform that connects the novel smart urban object adaptive park bench with an adaptive light system to actively support pedestrians in the urban environment, in particular senior citizens with handicaps.
\end{abstract}

\section{INTRODUCTION}

Internet of things (IoT) technology is increasingly used to make the urban environment "smart". With IoT-enabled process control and allocation mechanisms, to date many innovations aim at new possibilities for more effective traffic load balancing [1], [2], energy supply [3], [4], or waste management [5], [6]. While these innovations address important ecological problems the potentials to support pedestrians in the urban environment with IoT enabled physical urban objects are still in its infancy.

IoT technology connects physical objects via digital information processing and communication technology. By further equipping urban objects with sensors and actuators the urban environment is transformed into a cyber physical system [7] providing advanced potentials for pedestrian support. To make use of these potentials we design smart urban objects (SUO's) that adapt individually to pedestrians' specific requirements for the usability of the urban environment. Connecting SUOs with IoT allows for useful interactions between both, the objects with pedestrians, and the objects with each other.

While SUOs are supposed to improve usability for every pedestrian, a special focus is on pedestrians with handicaps. For handicapped pedestrians, the adaptivity of the SUOs shall not only enhance usability but effectively compensate (some of) their handicaps and thus enable safe usage of the urban space. A growing group of stakeholders who are in need for an adaptive and supportive urban environment are older adults
[8]. Hence "smartification" of the urban environment to meet pedestrians' needs is not only a technical gadget but plays a crucial societal role.

This research contributes to the knowledge base of designoriented research on the application of IoT technology in supportive urban environments to shape the smart city of the future. To that end, we provide a conceptual design of an IoT platform that connects the novel SUO adaptive adaptive park bench with an innovative adaptive light system. The leading question is: How to design the SUOs and their connection via IoT to enable supportive interactions between the objects and pedestrians for enhancing safe use of the environment?

The paper proceeds as follows: In the next section we briefly present recent developments within the smart city paradigm, IoT technology, smart street lighting and smart park benches. Then we report on our design of an IoT platform and the two SUOs adaptive park bench and adaptive light. Thereafter, we discuss a scenario for an interplay of the two SUOs, the IoT platform and pedestrians. We conclude and provide an outlook on future research.

\section{State of THE ART}

\section{A. The Smart City Paradigm}

For the characterization of a smart city, several perspectives play a role: Technology innovations, organizational innovations and policy innovations [9]. While technology innovations seem to be only one part among three, they are essential for all innovations within the smart city paradigm: Organizational innovations are directly related to an effective use of technology and policies shall actively promote the use of effective technology innovations [9].

An overall objective of smart city innovations is sustainability, economic growth and quality of life [10]. Current trends in smart city developments involve the domains of natural resources and energy, transport and mobility, buildings, living, government, as well as economy and people [11].

From a design-oriented perspective, we contend that information technological innovations provide most effective means to enhance smartness of cities in particular with respect 
to quality of life. Smart city initiatives use high technology innovations, such as IoT, to create benefits for well-being, inclusion and participation [12]. In conclusion, smart city relates to socio-technological developments for a harmony of material and virtual world [9]. Information technology plays an important role in contributing to solutions for emerging problems of urban living [11].

The information technological foundation to bring smart city innovations into built infrastructure are smart objects [13]. Smart objects in the urban area provide sensor data that can be used for urban environment monitoring, social networking, intelligent transport systems [14], or other information-intensive urban management problems. The aim of our research, however, is to go beyond exploiting smart objects as sensor networks. Our proposed SUOs have actuators and create benefits by actively adapting to citizens in interconnected scenarios.

\section{B. Internet of Things Platforms}

In the field of IoT architectures, many frameworks, models and platforms have already been developed seeking to solve both, specific concerns and general problems [15]. Nevertheless, there is still a significant lack of IoT middleware, which can be adapted to specific domains as well as to general applications [15], [16]. While one of our aims is to enhance safety of the users who indirectly use the IoT platform in the urban environment, we identify that existing solutions focus only pure technical aspects of safety and security [17]. A bridge to safety of IoT system users is rarely provided and is an essential point of research. A proposal for a solution for an IoT architecture that focuses on the problems describend in [15], [16] and thus provides a suitable basis for further developments is provided in [18]. Although in [18] security aspects of the solution play a role at the technical level, they are not specifically considered on the user level.

\section{Smart Urban Objects}

1) Smart Park Benches: Although seldom recognized as these, park benches are one of the most ubiquitous elements in public space [19]. By park bench we denote seats in the public area dedicated for more than one person. In urban life those seats play an important role for well-being. Yet, the idea of enabling park benches for adaptivity has not been widespread. From the perspective of user requirements adaptivity of park benches would provide clear gains for its usability. In practice, there are so called smart park benches which are supplied by solar energy and provide USB- and charging slots for smartphones, $\mathrm{WiFi}$, and environmental sensor equipment [20], [21]. However, these park benches do not have moving actuators but at best screens to display information. There are further ideas to augment public seats with information technology but these are rather for entertainment purposes than for usability purposes [19].

In designing park benches, knowledge about usability has long been incorporated, even though in a static manner. In a comprehensive study on design principles that shall make park benches particularly usable for senior citizens, it has been surveyed based on focus-groups and expert interviews, that seniors require elevated sitting positions, slightly tilted forward seats, and armrests but footrests should be avoided due to tripping hazard [22]. While there are several vendors of park benches with these special characteristics, as a matter of fact static alignment to these requirements can only account for the average but not for the individual. The most adaptive type of park benches, which are available, are those with pneumatic springs [23]. The opportunities of making park benches adaptive is up to now exploited only hardly.

2) Smart Street Lighting: In the past, many different concepts have been presented that include an intelligent lighting system. Several objectives have been addressed and several milestones have been achieved.

Motivated by using natural light more efficiently in buildings and consequently to reduce the energy consumption of the electrically generated light, a lighting system has been presented in order to meet this challenge [24]. The intelligence consists of a sensor-based detection of the available daylight inside the facility, whereupon the artificially generated light is regulated.

In addition to the lighting systematics within a building [24], there exist achievements presented in [25], [26], [27] that augment street lighting with intelligence. The light intensity is adapted depending on the traffic situation. The overriding objective of these efforts is to minimize the required energy consumption. Networking of the individual lamps already exists in [25]. The user's view of smart lighting systems is examined in [28], [29]. The focus here is on the psychological effect of different light modes on the user. Particularly in [28], the mood of older people is positively influenced by different lighting configurations. However, the core of the investigations here lies more on psychological mood analyses than on the concept of a smart lighting system.

\section{Design of Interconnected SMart URban OBJECTS}

\section{A. Internet of Things Platform}

To coordinate all SUOs in the overall system, we use an IoT platform. The platform is based on IoT technology and is also the central unit for processing joint information. The platform is designed against the following requirements: It should

1) be expandable with respect to SUOs and other IoT devices,

2) facilitate customization and connection of processes,

3) support and use standards,

4) exhibit little latency for a tactile internet,

5) be secure and guard privacy and user safety,

6) apply intelligent algorithms on interaction log data.

(1.) Expandability is required to add potentially new SUOs or IoT devices. Therefore, open interfaces, protocols and data formats are relevant.

(2.) As different users of SUOs may have different demands for the administration and behavior of the SUOs, the underlying processes must be determined flexibly. Also, the platform 


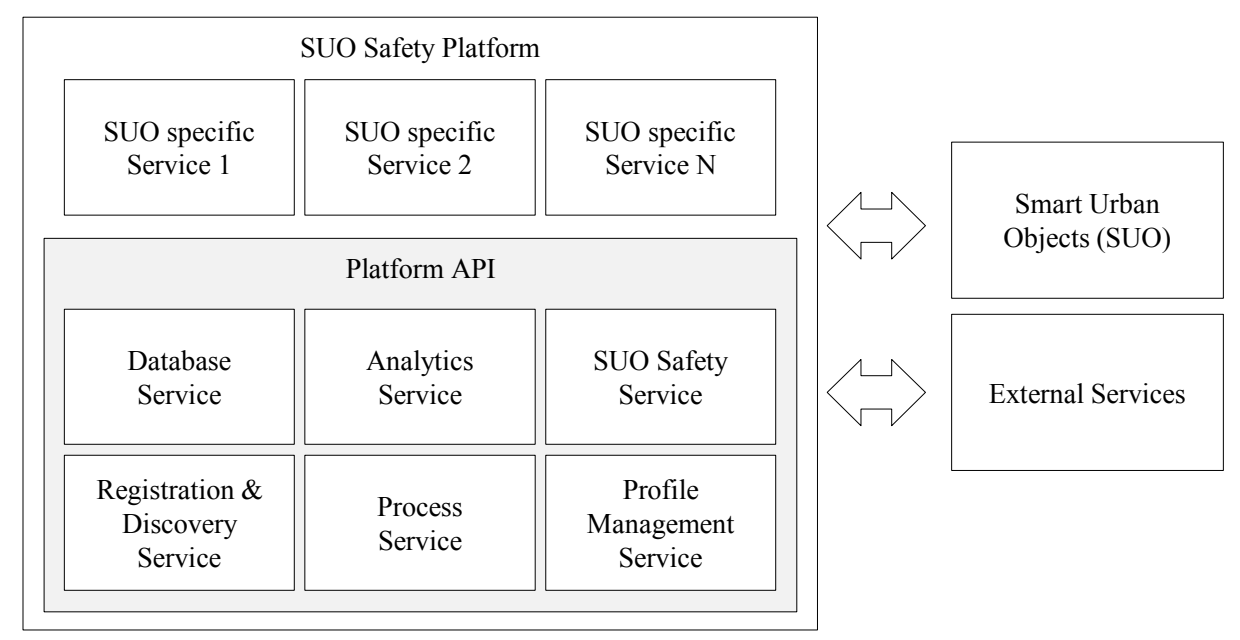

Fig. 1. Overview of the architectural design for the IoT platform

shall facilitate to combine any SUO, independently from the specifics of the SUO.

(3.) To meet the aforementioned requirements standardized interfaces and communication technology are prerequisite.

(4.) The platform is also a middleware for SUOcommunication. High latencies of control messages within the SUO-network may be disadvantageous or even harmful because the SUOs are situated in public spaces.

(5.) Communicated data must be guarded against deletion, manipulation, and unauthorized reading. The functionality must be reliable and save.

(6.) Generated and collected data shall be analyzed and utilized for improving the service provision for the end users.

Based on these requirements and the challenges identified in [18] we design the architecture of our IoT platform as depicted in figure 1 .

Basically, the platform contains two parts. The first part is the API part. There, those services run which must be available to the other, more specific services. The second part is for the specifics of the SUOs. There, those services run which are specific for single SUO or particular groups of SUOs. Arbitrary external services can be connected to the platform, too, as long as they use the supported protocol standards.

In the API part different basic services run to realize the core functionality of the IoT platform. This entails a central service for data storage. So all services can use a separate data storage according to the microservice pattern but can access these with a joint interface.

To connect and customize processes with SUOs the platform provides a processing service. Processes can be modeled with BPMN (Business Process Modeling and Notation) and are executed with a process engine. A process is a sequence of services that link several API and SUO-services. An analytics service can be integrated to enable descriptive, predictive, and prescriptive analyzes.

To ensure reliability and safety for end users in the public space, the SUO safety service provides required information based on user profiles and interaction models. To allow SUOspecific services to access SUO-data and user data the registration and profile management service provide a central interface to obtain user information and SUO meta-data.

Focusing the requirement for low latency, expandability and security several well-known protocols and technologies are used. Communication between platform and SUOs as well as external services is standardized with REST respectively MQTT interfaces. Both are encrypted. For platform internal communication either the HTTP/2 protocol (REST) can be used or for particularly critical services with respect to latency a gRPC interface with protocol buffers can be used. Data are stored in a datalake on the platform. The services run with container based orchestration to account for high flexibility.

\section{B. Adaptive Park Bench}

The perception of the usability of park benches varies among people. Thereby, a crucial factor is the extent to which the design and functionality of a park bench fits the individual anthropometric measures. The designs of conventional park benches constitute, due to their static characteristics, necessarily always a compromise between the divergent requirements between the variety of users. Although in everyday life citizens rarely notice imperfectness of park benches, enhanced individual alignment in design and functionality is expected to make a significant difference. When focusing people with infringed constitution, the present imperfectness may even be an effective impediment for usage.

Anthropometric studies provide, for instance, evidence that differences exist in relevant measures between senior citizens, aged 65 or more, and younger citizens [30], [31]. Aligning seat height to individual measures is one way to increase ergonomics, i.e. usability. However, this is only effective while sitting. For people with weakened musculature, and limitations to hold the balance the process of sitting down and standing up is a serious source of difficulties. And here, a contradiction arises which cannot be resolved by static design: For standing 


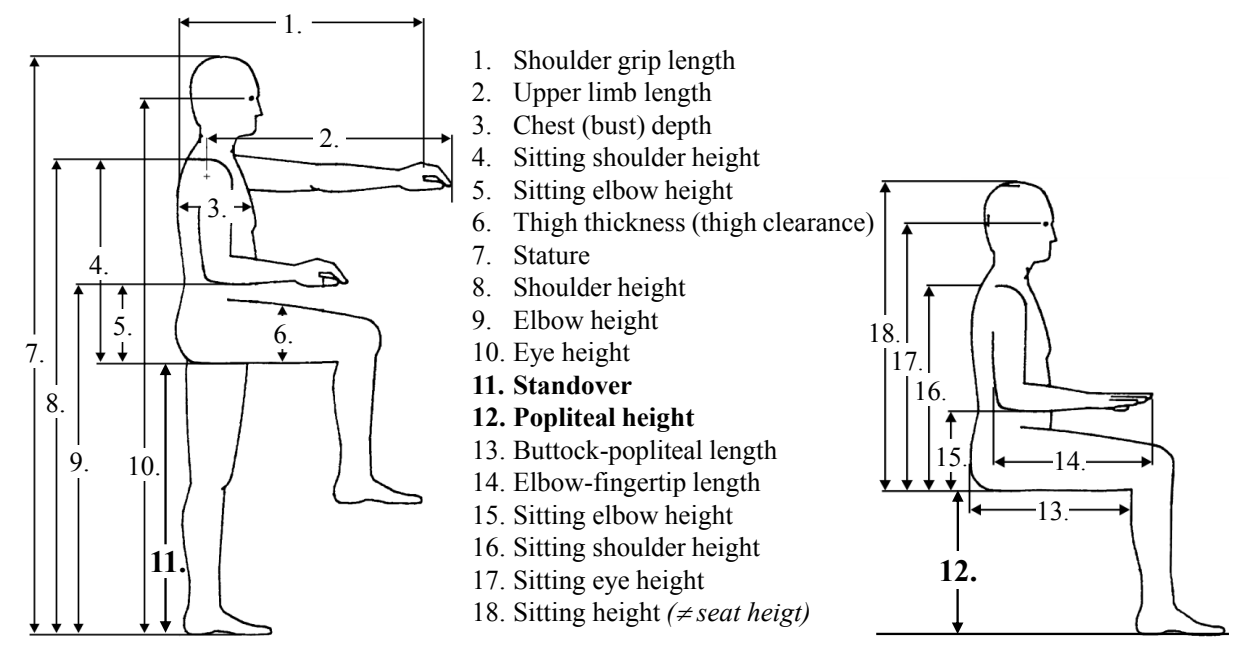

Fig. 2. Anthropometric measures, adopted from [30]; relevant for the adaptive park bench are 11. standover and 12. popliteal height.

\begin{tabular}{|c|c|c|}
\hline & \multicolumn{2}{|l|}{ ParkbenchUser } \\
\hline & \begin{tabular}{|l|} 
Attribute \\
\end{tabular} & Datatype \\
\hline \multirow{8}{*}{ core data \{} & id & String \\
\hline & age & Integer \\
\hline & authorizationGroup & String \\
\hline & authorized & Boolean \\
\hline & standover $[\mathrm{mm}]$ & Integer \\
\hline & poplitealHeight $[\mathrm{mm}]$ & Integer \\
\hline & sittingElbowHeight [mm] & Integer \\
\hline & maxDistanceUntilRestIsAdvised [m] & Integer \\
\hline cess data $\prec$ & distanceSinceLastRest [m] & Integer \\
\hline
\end{tabular}

Fig. 3. Datamodel for a park bench user.

up and sitting down, park benches with elevated seat height are easier to use but while sitting they are unergonomic. The same type of contradiction applies to the tilt of the seat. A seat which is tilted forwards facilitates gliding out of the seating position and eases standing up. While sitting, however, a slight tilt backwards is ergonomic. The chance with making park benches adaptive is not only that they are enabled to align to various but static measures. It also allows for a novel type of support for citizens. Active support by the urban environment does not only enhance its usability but makes it also a safer space for pedestrians with handicaps. Among the anthropometric measures depicted in figure 2 the relevant ones, to which the bench shall adapt, are 11. standover and 12. popliteal height.

To reduce difficulties with standing up and sitting down the park bench adjusts it seat height and seat tilt according to the respective phase: At the beginning of sitting down the seat is elevated and tilted forwards. Then it smoothly descends and tilts backwards for ergonomic sitting. For standing up the procedure is just reverse. When a person who needs support with sitting down to a seat approaches the adaptive park bench, the bench, first aligns its seat height to the standover of this person and tilts forwards. After the person has sat or leaned on the elevated seating surface the bench smoothly adjusts the surface to the person's popliteal height and tilts backwards. When the person wishes to stand up the bench slowly tilts forwards and elevates up to the standover again. For persons who do not need support in sitting down and standing up the first and last phase may be simply omitted or reduced in its amplitude (that is: the bench does not elevate up until the standover but only a little). To detect a person, who approaches the bench, short distance radio technology can be used, such as Bluetooth. People carry a respective portable device (which is also required for the adaptive light system). To clearly detect that an approaching person actually wishes to sit down a "manual" interaction is required. The simplest way is to attach a button to the bench or to send a corresponding signal via the required portable device. If no manual interaction is used to indicate the wish to sit down, but a weight sensor recognizing persons sitting on the seat 


\begin{tabular}{|l|l|}
\hline \multicolumn{2}{|c|}{ AdaptiveParkbench } \\
\hline Attribute & Datatype \\
\hline id data & Integer \\
\hline geoLocation & Array of Real [3] \\
\hline widthOfSeat [mm] & Integer \\
\hline depthOfSeat [mm] & Integer \\
\hline amountOfSeparateSections & Integer \\
\hline amountOfArmrests & Integer \\
\hline isSeatHeightAdaptive & Boolean \\
\hline isSeatTiltAdaptive & Boolean \\
\hline isArmrestHeightAdaptive & Boolean \\
\hline surfaceMaterial & Enum [timber, plastic metal, stone] \\
\hline backrestMaterial & Enum [na, timber, plastic, metal, stone] \\
\hline currentSeatHeight [mm] & Integer \\
\hline currentArmrestHeight [mm] & Integer \\
\hline currentSeatTilt [degree] & Integer \\
\hline numberOfSittingPersons & Integer \\
\hline
\end{tabular}

Fig. 4. Datamodel for an adaptive park bench.

surface then the bench can automatically adapt to the person's popliteal height (always assumed that the person carries the device with the required data). The "smartest" way, however, would be to infer that a person must be wishing to sit down. Figure 3 outlines the data structure of a park bench user required for executing the supportive function of the adaptive park bench and figure 4 outlines the data structure for the adaptive park bench.

\section{Adaptive Light}

As pointed out previously, there is up to now no street lighting system that implements an adaptive lighting configuration depending on individual positions of pedestrians. Therefore we are designing a concept for realizing a smart lighting system and present basic requirements. In section IV we discuss how these basic requirements relate to the overall system.

The adaptive light system shall be aligned to increase wellbeing of citizens. A particular goal is to enhance participation in urban life for older adults. The adaptive lighting system is therefore used as a means to induce positive impact on well-being in the urban area and to stimulate participation by varying light intensity or color.

As a use case we employ routing to specific points of interest by means of varying light colors and intensity and derive the following basic requirements:

1) Street lamps must be interconnected and be able to communicate with each other via the interconnection technology.

2) The person to whom the lights adapt must be identified within this interconnection structure (same technology as for the identification of park bench users).
3) Information exchange between the person an the light system must be available.

4) The lights must be able to change color and intensity.

\section{SCENARIO FOR INTERCONNECTED SMART URBAN OBJECTS}

Figure 5 sketches a scenario to demonstrate the usefulness of interconnecting the SUOs. Figures $6-8$ show sequence diagrams for interactions of an SUO with the IoT platform and a user by the example of an adaptive park bench. The sequences sit down and stand up (figures $7+8$ ) are specific interactions with the park bench and replace the interaction placeholder in figure 6 for the example case that the SUO is an adaptive park bench. For other SUOs the respective interaction is inserted there. For adaptive light, for instance, the interaction could be that the light changes intensity or color for a pedestrian. Note, that a ParkbenchUser is meant to be extending the general SUO user class Pedestrian by containing additionally the specific attributes required for the park bench interactions.

Figure 6 outlines the intertwined logic between SUOs and the platform. SUOs interact locally with their users but the platform permanently monitors the SUOs and periodically stores the monitored states as the respective SUO data objects. These enable data analytics on interactions. Beyond that, data of several SUOs can be jointly analyzed, the platform facilitates data exchange between SUOs and can actively start control sequences for the SUOs. We now describe a scenario where the two example SUOs interact with each other and generate a benefit for a pedestrian. 


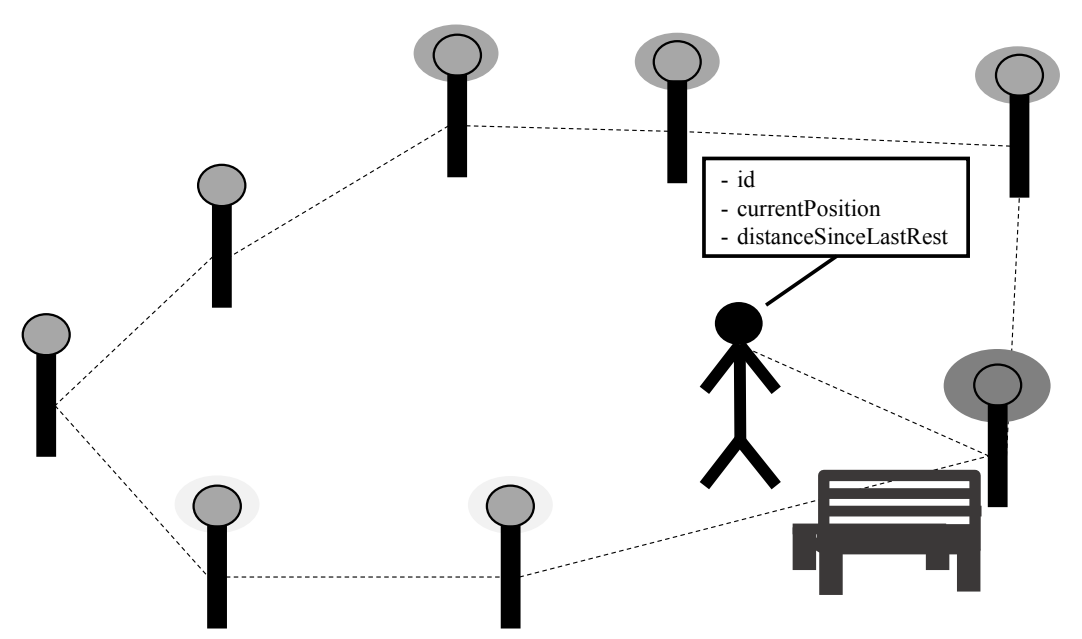

Fig. 5. Interconnected system of adaptive lighting and adaptive park bench

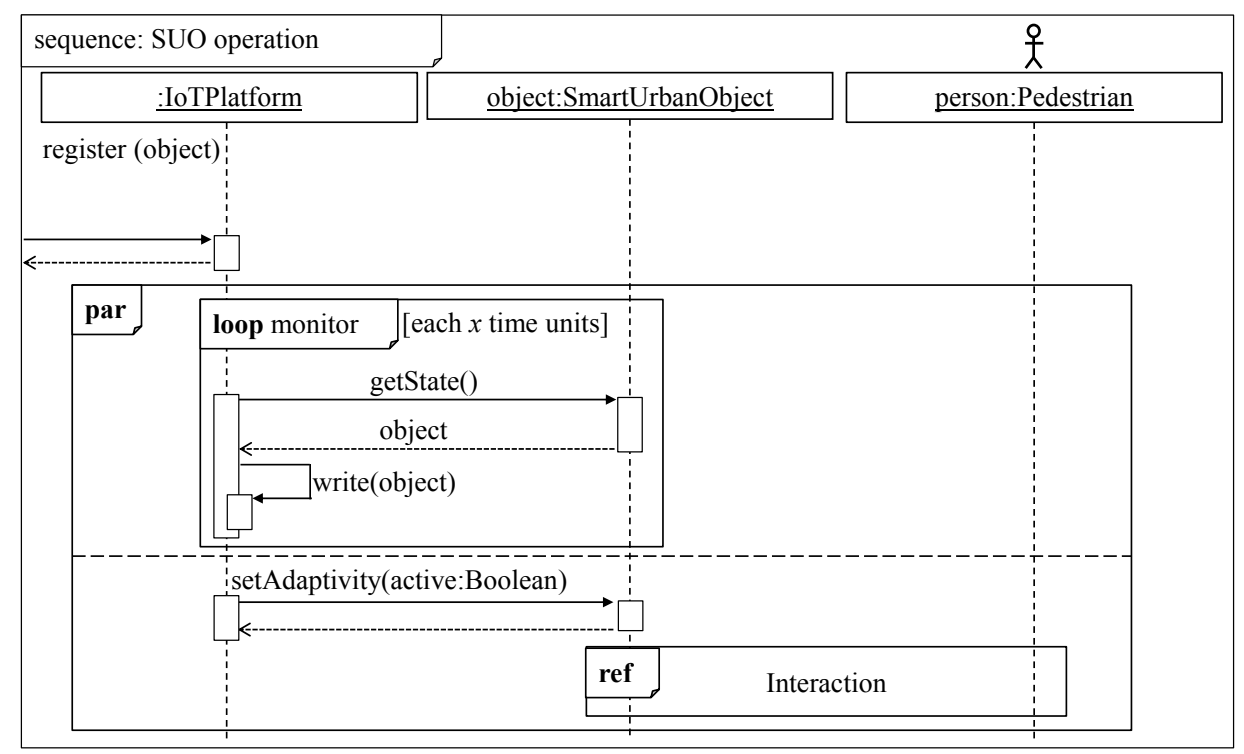

Fig. 6. Sequence diagram for operating SUOs.

The SUOs of this scenario are the adaptive park bench and the adaptive light system. The user is an older adult who needs to rest from time to time. ${ }^{1}$ The environment is a pedestrian area which is equipped with adaptive park benches and adaptive lights. The benches are installed every 100 meters, the light posts have a spacing of 25 meters to each other, and they are arranged in such a way that at each park bench, there is a light. As a matter of fact, park benches are sometimes occupied, when needed. This may be indeed a severe problem for older adults needing a rest. In the presented scenario, however, the adaptive lights and park benches may help reducing this problem.

The IoT platform records the interactions of the older adult with the adaptive park bench. So the system knows when the user had his last rest. The adaptive light posts recognize

\footnotetext{
${ }^{1}$ Depending on the constitution this may be even the case every 300 meters.
}

when the person passes so the IoT platform can automatically infer the distance since the person's last rest, too. Having information about the distances after which the older adult is recommended to take a rest, the smart city infrastructure can take actions to maintain a high well-being of this citizen. Indirectly, the system cares for the safety of the older adult.

If an older adult needs a rest in the next time, then the smart city system changes the colors of the lights at reachable and free benches. If the next free park bench is farther away then the lights increase their intensity so the older adult can see the hint to a free park bench from far. While approaching the park bench, which is traceable via the lighting system, the bench adjusts its seat height already to the respective anthropometric measure of this person so that the person can directly sit down comfortably. Position tracking with the light posts promises a higher precision of position determination than using standard 


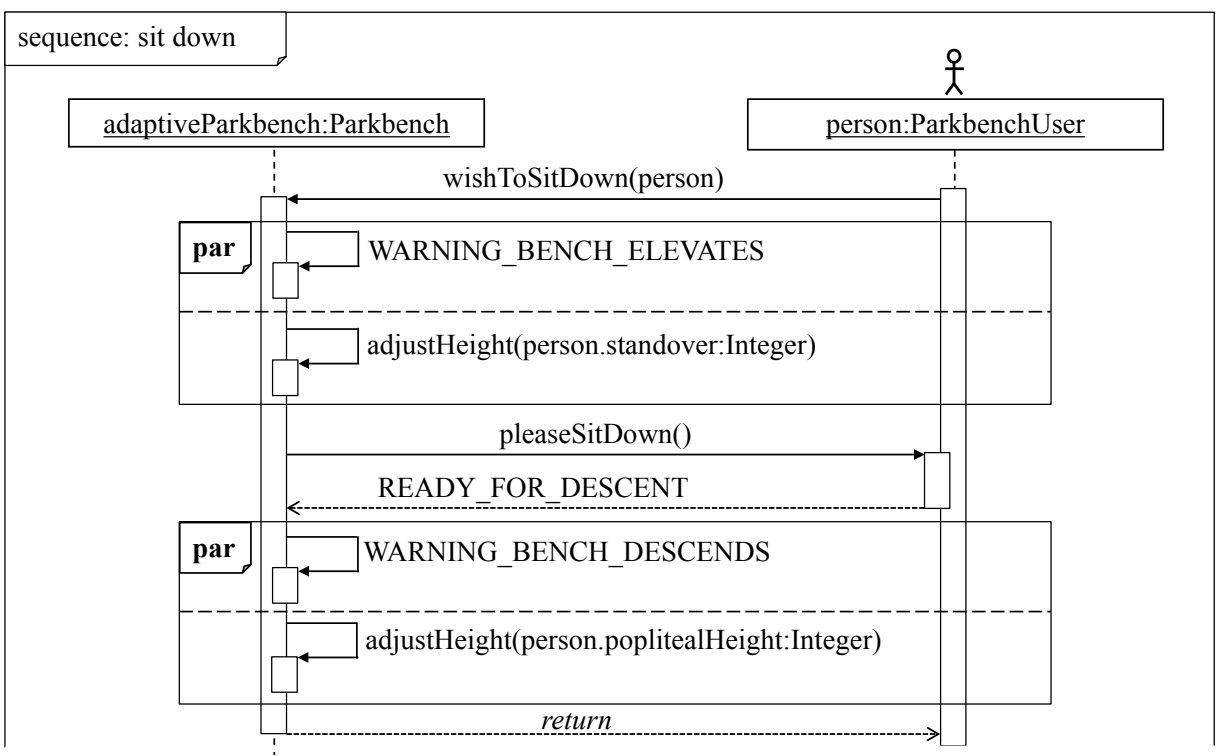

Fig. 7. Sequence diagram for sitting down with support of the adaptive parkbench.

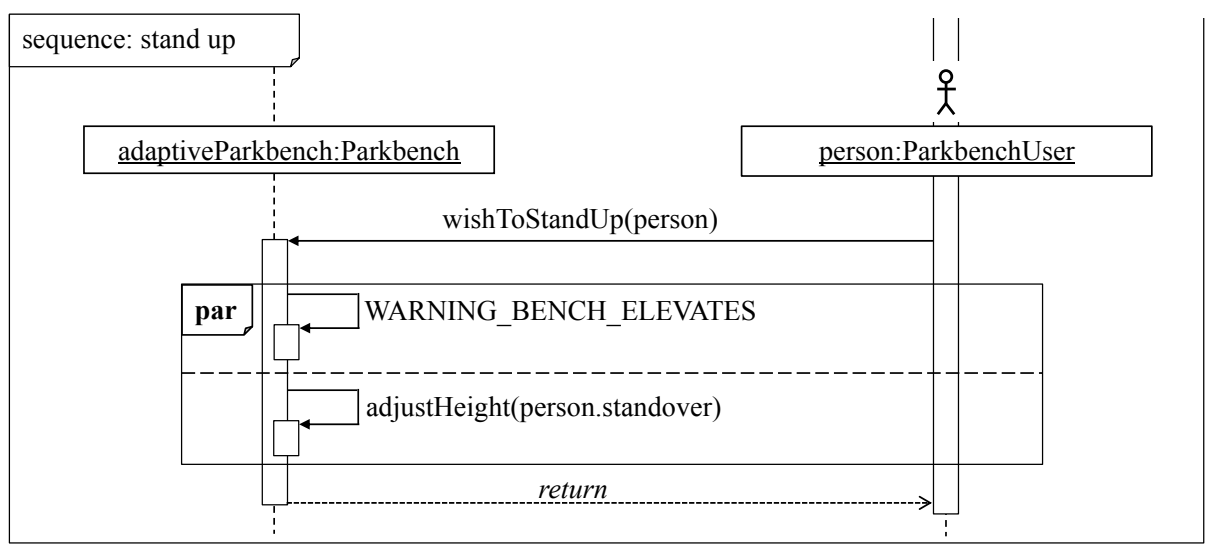

Fig. 8. Sequence diagram for standing up with support of the adaptive parkbench.

GPS alone.

More differentiated support by the smart infrastructure if multiple users need a rest at the same time in the same area is subject to our ongoing research (for the two-user case an adaption rule for simultaneous use of adaptive park bench has been recently studied in [32]).

\section{CONCLUSIONS AND OUTLOOK}

We presented designs for SUOs, particularly to facilitate urban life for people with handicaps. As SUOs adapt to personal needs, they are likely to enhance urban life for all citizens. We discussed a scenario where SUOs provide additional benefits for a smart city by interconnecting them with an IoT platform. The IoT platform serves as middleware and provides services to maintain various SUOs. At the same time the platform gathers data from the SUO for the purpose of analyzes. The inferred information are expected to be an essential factor to make modern cities and urban life smarter.
We are aware of privacy issues and societal risks that may occur in environments that digitize individuals. However, research suggests that IT-based services for the urban area can support in counteracting age-related decline of outdoor activities [33], [34]. Our concern is focused on employing SUOs to provide active IT-based support. One of the benefits for which we aim is enhanced safety for individuals in urban environments. To this end, we will further explore which hazards are relevant in citizens everyday activities and how we can mitigate them with interaction and interconnection between smart urban objects and citizens.

\section{ACKNOWLEDGEMENT}

This work is has been part of the research project URBANLIFE+, supported by the Federal Ministry of Education and Research, Germany, under grants 16SV7438K and 16SV7442. 


\section{REFERENCES}

[1] Cheng, Z. (A.), Pang, M.-S., Pavlou, P. A.: On Intelligent Transportation Systems and Road Congestion. In: 37th International Conference on Information Systems (ICIS 2016), Dublin, Ireland.

[2] Li, Z., Hong, Y., Zhang, Z.: An empirical analysis of on-demand ride sharing and traffic congestion. In: 37th International Conference on Information Systems (ICIS 2016), Dublin, Ireland.

[3] Fridgen, G., Häfner, L., König, C. Sachs, T.: The Value of IS-Enabled Flexibility in Electricity Demand - a Real Options Approach. In: 12th International Conference on Wirtschaftsinformatik (WI 2015), Osnabrück, Germany, 993-1007.

[4] Wanger, S., Brandt, T., Neumann, D.: IS-Centric Business Models for a Sustainable Economy - The Case of Electric Vehicles as Energy Storage. In: 12th International Conference on Wirtschaftsinformatik (WI 2015), Osnabrück, Germany, pp. 1055-1070.

[5] Chowdhury, B., Chowdhury, M. U.: RFID-based Real-time Smart Waste Management System. In: Australasian Telecommunication Networks and Applications Conference 2007 (ATNAC 2007), pp. 175-180. Institute of Electrical and Electronics Engineers, Inc (IEEE), Piscataway, New Jersey.

[6] Glouche, Y., Couderc, P.: A Smart Waste Management with SelfDescribing objects. In: 2nd International Conference on Smart Systems, Devices and Technologies (SMART 2013), pp. 63-70.

[7] Wu, F.-J., Kao, Y.-F., Tseng, Y.-C.: From wireless sensor networks towards cyber physical systems. Pervasive and Mobile Computing 7, 397-413 (2011).

[8] Haustein, T., Mischke, J., Schönfeld, F., Willand, I. (eds.): Older people in Germany and the EU. Federal Statistical Office, Wiesbaden, Germany (publ.), 2016.

[9] Nam, T., Pardo, T. A.: Smart City as Urban Innovation: Focusing on Management, Policy, and Context. In: Proceedings of the 5th International Conference on Theory and Practice of Electronic Governance (ICEGOV 2011), 185-194. ACM (2011).

[10] Caragliu, A., Del Bo., C., Nijkamp, P.: Smart Cities in Europe. Journal of Urban Technology 18:2, 65-82 (2011).

[11] Neirotti, P., De Marco, A., Cagliono, A. C., Mangano, G., Scorrano, F.: Current trends in Smart City initiatives: Some stylised facts. Cities 38, 25-36 (2014).

[12] Dameri, R. P.: Searching for Smart City definition: a comprehensive proposal. International Journal of Computers \& Technology 11:5, 25442551 (2013).

[13] Chourabi, H., Nam, T., Walker, S., Gil-Garcia, J. R., Mellouli, S., Nahon, K., Pardo T. A., Scholl, H. J.: Understanding Smart Cities: An Integrative Framework. In: Proceedings of the 45th Hawaii International Conference on System Sciences (HICSS 2012), 2289-2297, IEEE.

[14] Vasseur, J.-P., Dunkels, A.: Smart cities and urban networks. In: Interconnecting Smart Objects with IP: The Next Internet, ch. 22, pp. 335-352, Morgan Kaufmann, Burlington (2010).

[15] Mineraud, J., Mazhelis, O., Su, X., Tarkoma, S.: A gap analysis of Internet-of-Things platforms. Computer Communications, 89, 5-16 (2016).

[16] Razzaque, M. A., Milojevic-Jevric, M., Palade, A., Clarke, S.: Middleware for internet of things: a survey. IEEE Internet of Things Journal 3(1), 70-95 (2016).

[17] Vasilomanolakis, E., Daubert, J., Luthra, M., Gazis, V., Wiesmaier, A., Kikiras, P.: On the Security and Privacy of Internet of Things Architectures and Systems. In: Proceedings of the International Workshop on Secure Internet of Things (SIoT 2015), 49-57, IEEE.
[18] Wehlitz, R., Häberlein, D., Zschörnig, T., Franczyk, B.: A Smart Energy Platform for the Internet of Things - Motivation, Challenges, and Solution Proposal. In: Abramowicz W. (eds.) Business Information Systems (BIS). Lecture Notes in Business Information Processing 288. Springer, Cham (2017).

[19] Dekel, A., Simon, Y., Dar, H., Tarazi, E., Rabinowitz, O., Sterman, Y.: Adding Playful Interaction to Public Spaces. In: Intelligent Technologies for Interactive Entertainment. Springer, Berlin, Heidelberg, 225229 (2005).

[20] https://www.3fficient.com/shop/solar-smart-bench/, last accessed: 201807-18.

[21] http://www.soofa.co/getsoofa/ , last accessed: 2018-07-18.

[22] Beucker, N., Zurnatzis, M.: Stadtmobiliar für Senioren. Ausstattungskriterien für eine altengerechte Stadt [Urban furniture for senior citizens. Configuration criteria for an elderly-friendly city]. Technical Report, University of Applied Sciences Niederrhein, Germany (2011).

[23] https://abes-online.com/en/product/bench-1-110-1/ last accessed: 2018$07-18$.

[24] Nippuni Kumaar, A. A., Kiran, G., Sudarshan, T. S. B.: Intelligent Lighting System Using Wireless Sensor Networks. Int. Journ. of Ad hoc., Sensor \& Ubiquitous Computing (IJASUC) 1:4, 17-27, (2010). doi:10.5121/ijasuc.2010.1402

[25] Crowther, J., Herzig, C., Feller, G.: The Time Is Right for Connected Public Lighting Within Smart Cities. Cisco Internet Business Solutions Group (IBSG) (2012)

[26] Escolar, S., Carretero, J., Marinescu, M., Chessa, S.: Estimating Energy Savings in Smart Street Lighting by Using an Adaptive Control System. International Journal of Distributed Sensor Networks, 117, (2014). doi:10.1155/2014/971587

[27] Jackson, H., Jackson, S., Jackson, C., Siminovitch, M.: Saving Energy in Buildings with Adaptive Lighting Solutions. California Energy Commission. California Lighting Technology Center, UC Davis (2015).

[28] Huldtgren, A., Katsimerou, C., Kuijsters, A., Redi, J. A., Heynderickx, I. E. J.: Design Considerations for Adaptive Lighting to Improve Seniors Mood. Inclusive Smart Cities and e-Health, 9102. In: Lecture Notes in Computer Science, 1526, Springer International Publishing (2015).

[29] Kuijsters, A., Redi, J., Ruyter, B. d., Heynderickx, I.: Lighting to Make You Feel Better: Improving the Mood of Elderly People with Affective Ambiences. PloS one 10:7 (2015). doi:10.1371/journal.pone.0132732

[30] Kothiyal, K., Tettey, S.: Antropometry for Design for the Elderly. Int Journ. of Occupational Safety and Ergonomics 7:1, 15-34 (2001).

[31] Adler, M., Herrmann, H.-J., Koldehoff, M., Meuser, V., Scheuer S., Müller-Arnecke, H., Windel, A., Bleyer, T.: Ergonomiekompendium: Anwendung ergonomischer Regeln und Prüfung der Gebrauchstauglichkeit von Produkten [Ergonomics compendium: Application of ergonomic rules and assessment of usability of products]. Federal Institute for Occupational Safety and Health (eds.), Germany (2010).

[32] Hubl, M.: Adaption Rule for Simultaneous Use of Smart Urban Objects from a Fairness Perspective. In: Proceedings of the 20th International Conference on Business Informatics (CBI 2018), 89-98, IEEE. doi:10.1109/CBI.2018.00019

[33] Fietkau, J.: The case for including senior citizens in the playable city. In: Proceedings of IEEE/WIC/ACM International Conference on Web Intelligence, Leipzig, Germany, August 2017 (WI'2017), 1072-1075. doi:10.1145/3106426.3109042

[34] Leukel, M., Schehl, B., Wallrafen, S., Hubl, M.: Impact of IT Use by Older Adults on Their Outdoor Activities. In: Proceedings of the 38th International Conference on Information Systems (ICIS 2017). 